\title{
Coordinated Perimeter Control for Multiregion Heterogeneous Networks Based on Optimized Transfer Flows
}

\author{
Yajuan Guo, ${ }^{1,2}$ Licai Yang $\mathbb{D}^{2},{ }^{2}$ and Jun $\mathrm{Gao}^{3}$ \\ ${ }^{1}$ School of Traffic and Logistics Engineering, Shandong Jiaotong University, Jinan 250357, China \\ ${ }^{2}$ School of Control Science and Engineering, Shandong University, Jinan 250061, China \\ ${ }^{3}$ Qingdao Hisense TransTech Co., Ltd., Qingdao 266071, China
}

Correspondence should be addressed to Licai Yang; yanglc@sdu.edu.cn

Received 14 November 2019; Revised 20 July 2020; Accepted 13 August 2020; Published 1 September 2020

Academic Editor: Mauro Gaggero

Copyright (C) 2020 Yajuan Guo et al. This is an open access article distributed under the Creative Commons Attribution License, which permits unrestricted use, distribution, and reproduction in any medium, provided the original work is properly cited.

Exploring efficient control strategies for heterogeneously congested urban networks remains a big research challenge. The theory of macroscopic fundamental diagram (MFD) provides a new perspective for network-wide congestion control decisions. This paper proposes a coordinated perimeter control strategy for multiregion heterogeneous networks based on optimized transfer flows. First, a two-layer network partitioning method is presented to capture spatial heterogeneity dynamics of urban networks. For this partition, traffic flow equilibrium model based on MFD and multiagent based hierarchical traffic management scheme are built. Then, an improved multinomial logit model is developed for deriving optimized transfer flows among multiple congested regions. A coordinated perimeter control strategy using model predictive control is further proposed, which is aimed at tracking desired accumulations of each congested region. As a case study, the proposed control strategy is applied to the downtown network of Jinan, China, using simulation analysis. The results demonstrate that it can achieve balanced network flow distribution and increased mobility.

\section{Introduction}

Regional and even global traffic congestion patterns have become the norm in large- and medium-sized cities. Realistic modeling and efficient traffic control of the large-scale congested urban networks are becoming increasingly important. Most of the existing urban traffic management strategies are based on complex physical modeling at link level and computationally expensive numerical solution algorithms. The network-wide implementation of these methods might face some difficulties in terms of real-time feasibility. Furthermore, traffic control strategies like SCATS [1] and SCOOT [2] act less efficiently when it comes to saturated traffic conditions. These deficiencies emphasize the need for developing network-wide traffic management schemes and control strategies based on aggregate-level network modeling, which can efficiently manage critical components of the city traffic and generate a more sustainable city.
Recent studies have demonstrated that macroscopic fundamental diagram (MFD) is a powerful modeling tool for network-level traffic management strategies, such as perimeter control [3-5], congestion pricing $[6,7]$, and route guidance $[8,9]$, as it provides aggregated relationships between macroscopic traffic variables and simplifies complexity of traffic flow dynamics $[10,11]$. However, these MFD-based macroscopic strategies might not be optimal or feasible under heterogeneously congested conditions, due to the fact that uneven congestion distribution can affect the scatter or even the existence of MFD [12, 13]. Essentially, homogeneous urban areas are able to exhibit well-defined MFDs, i.e., a unimodal low-scatter curve between network accumulation and trip completion flow [14]. Thus, understanding and modeling the dynamics of heterogeneity is beneficial to shed light on how to design smarter traffic management methods for large-scale heterogeneous urban network. Saeedmanesh and Geroliminis [15] developed a static clustering algorithm to partition heterogeneous 
networks into connected homogeneous regions based on "Snake" similarities. Later, they extended the aforementioned method to dynamic framework by incorporating the spatiotemporal characteristics of congestion propagation [16]. In addition, the work in [17] proposed a fast network division approach based on correlation degree between two adjacent intersections. These partition results above ensure spatial connectivity and homogeneity of each region and provide a solid foundation for the application of MFD theory. But the regions of these partitioning networks are spatially closely connected, which could not accurately capture heterogeneous dynamics of urban road networks with different levels of congestion, especially discrete congestion patterns in the space domain. More recently, Guo et al. [18] proposed a methodology to dynamically identify critical congestion warning regions from heterogeneous networks, which can provide reliable and accurate research objects for traffic congestion control strategies based on MFD. Multiple congested regions extracted by this method are not directly connected, and there is a block of uncongested boundary region among them. Such congestion pattern fits better with the situations that are frequently observed in largescale urban network. The multiregion setting still requires some effort to clearly understand how boundary intersections should be further divided and collaboratively managed. This study moves toward this direction.

Extensive studies on perimeter control strategies using the theory of MFD have been carried out. These works fully prove that gating actions can be employed against congestion or oversaturation of urban network critical parts. Different control approaches have been proposed to achieve the single-region perimeter control, such as proportionalintegral-type (PI) feedback control [19], robust control [20], and Bang-bang control [21], with the main purpose of maintaining regional accumulations around the desired points by limiting inflows along the perimeter. Comparatively speaking, perimeter control for multiregion system with MFD-based modeling, i.e., manipulating transfer flows between adjacent regions, is deemed to be a more challenging work. Wan et al. [22] designed an adaptive perimeter control scheme for urban traffic networks with multiple regions, considering time-varying state and input delays. An online adaptive optimization algorithm was utilized to enhance the performance of optimal multivariable PI feedback regulators for multiregion perimeter flow control [23]. And the work in Haddad and Mirkin [24] developed a distributed adaptive perimeter (DAP) control method for multiregion MFD systems to accommodate the model parameter uncertainties and the restrictions on available information. However, these multiregion perimeter control approaches ignore the frequent situation where multiple congested regions are spatially separated by a certain distance. In addition, Yildirimoglu et al. [25] investigated the effects of spatial congestion heterogeneity and route choice behavior on MFD modeling. Their findings revealed that the proposed advisory route guidance system can create conditions close to dynamic system optimum state. So, recent works also attempt to develop hybrid traffic control strategies for multiregion system by integrating perimeter control and aggregated route guidance. Yildirimoglu et al. [26] proposed a hierarchical traffic management system that consists of regional route guidance and subregional path assignment based on the MFD heterogeneous modeling (i.e., regionbased model and subregion-based model [27]). A networkwide model predictive control (MPC) scheme with integrated perimeter control and regional route guidance was developed to improve mobility in urban networks [28]. Unfortunately, these strategies are studied based on the idealized road network partitions. The application effect of real networks has not been tested by the detailed simulation experiments.

To overcome the aforementioned difficulties, a two-layer network partitioning method is first presented by extending our previous study [18], so as to better characterize spatial heterogeneity dynamics of urban networks with multiple centers of congestion. Based on the partition, we reformulate the multiregion macroscopic traffic flow dynamics using MFD and develop a hierarchical traffic management scheme based on multiagent technology. Second, a transfer flow optimization model of multiple congested regions is developed based on multinomial logit (MNL) model, considering real-time accumulation states and spatial distances of adjacent regions. Third, a coordinated perimeter control strategy based on optimized transfer flows is proposed to operate urban network system close to dynamic traffic equilibrium.

The remainder of the paper is structured as follows. Section 2 extends a two-layer partitioning method for heterogeneous urban networks and presents aggregated modeling of traffic flow dynamics using MFD. In Section 3, we elaborate on multiagent based hierarchical traffic management scheme. In this framework, multiregion transfer flow optimization model and coordinated perimeter control strategy are further developed. Finally, multiregion perimeter control method is applied to the downtown network of Jinan City in Section 4. The last section concludes this paper and identifies future research directions.

\section{Partitioning and Modeling of Multiregion Heterogeneous Networks}

2.1. Two-Layer Partitioning of Heterogeneous Urban Networks. Traffic patterns in the real-life urban transportation network are typically heterogeneous due to the existence of different traffic demands and roadway facilities. And the distribution of traffic congestion is uneven and discontinuous in both temporal and spatial dimensions. Recent findings show that heterogeneous congested networks exhibit a MFD curve with high scatter, which causes uncertainties and inaccuracies for modeling of traffic dynamics. Hence, partitioning and modeling for heterogeneous congested urban road networks is an essential prerequisite for the design of MFD-based traffic control strategies [29]. The perimeter control of multiple congested regions studied in this paper is mainly based on the concept of two-layer network partitioning, including the extraction of homogeneous congested regions and the subdivision of noncongested boundary region. This detailed representation can provide more information on heterogeneous network traffic state. 
In fact, congestion always propagates with some finite speed in time and space dimension, and the adjacent road links are more likely to be congested than farther links. This spatial correlation of traffic congestion is fully utilized to extract a small number of homogeneous congested regions from heterogeneous urban traffic network, namely, firstlayer division. We have done this work using a community detection method, which was initially introduced in [18]. Each critical homogeneous congested region has spatial compactness and minimization of a heterogeneity metric (i.e., link traffic state parameter). These properties ensure that a well-defined MFD shape can be observed in each congested region, as shown in Figure 1(a). In most cases, multiple congested regions of road network exist in a spatial form that is not connected to each other, and there is a block of uncongested boundary region among them, which is the gray part in Figure 1(a). To better manipulate transfer flows of multiple regions at different levels of congestion, it is particularly important to subdivide uncongested boundary region after the first-layer division. This second-layer division is depicted in Figure 1(b) where $B_{a, b}$ is boundary region between adjacent congested regions $a$ and $b, a, b \in\{i, j, l, h\}$ and $a \neq b$. Taking Figures 1(a) and 1(b) as an example, the two-layer partitioning algorithm is briefly summarized in Algorithm 1.

\subsection{Traffic Flow Equilibrium Model for Multiregion Systems.} Let us assume that an urban network is partitioned into homogeneous congested region set $W$ with relatively perfect MFDs and boundary region set $S$. Among them, congested regions are with a high degree of saturation and prone to traffic congestion. We first discretize time into equally spaced intervals, namely, $t=k \cdot T$, where $k=1,2,3, \ldots, K$ and $T$ is simulation step. Thus, the simple conservation equation $[23,30]$ for each congested region $i$, $i \in W$, is presented to capture the dynamic evolution of traffic system as follows:

$$
n_{i}(k+1)=n_{i}(k)+T\left(Q_{i, \text { in }}(k)-Q_{i, \text { out }}(k)\right),
$$

where $n_{i}(k)$ (veh) is the total accumulation (number of vehicles) in region $i$ during $k$ th period; $Q_{i, \text { in }}(k)$ and $Q_{i, \text { out }}(k)$ (veh/s) are the total inflow and outflow in region $i$ during $k$ th period, respectively. The total inflow to region $i$ is given by

$$
Q_{i, \text { in }}(k)=q_{i, \text { ins }}^{\text {in }}(k)+q_{i, \text { ext }}^{\text {in }}(k)+\sum_{j \in W_{i}} \widehat{m}_{j i}(k),
$$

where $q_{i, \text { ins }}^{\text {in }}(k)(\mathrm{veh} / \mathrm{s})$ is the internal traffic demand generated in region $i$ during $k$ th period; $q_{i, \text { ext }}^{\text {in }}(k)(v e h / s)$ is the inflow to region $i$ from neighbouring external region during $k$ th period; $\widehat{m}_{j i}(k)(\mathrm{veh} / \mathrm{s})$ is the effective transfer flow from neighbouring congested region $j$ to $i$ through boundary region $B_{i, j}$ at time step $k$; $W_{i}$ is the set of neighbouring congested regions that are reachable from region $i$ through boundary regions.

Also, $Q_{i, \text { out }}(k)$ is the sum of the effective transfer flows from congested region $i$ to all the neighbouring congested regions (i.e., $\left.\sum_{j \in W_{i}} \widehat{m}_{i j}(k)\right)$ and the outflow to neighbouring external region (i.e., $\left.q_{i, \text { ext }}^{\text {out }}(k)(\mathrm{veh} / \mathrm{s})\right)$, plus the internal trips completed within region $i$ (i.e., $\left.q_{i, \text { ins }}^{\text {out }}(k)(\mathrm{veh} / \mathrm{s})\right)$. The term $Q_{i, \text { out }}(k)$ can be given by the output $G_{i}\left(n_{i}(k)\right)(\mathrm{veh} / \mathrm{s})$ (the total trip completion rate in region $i$ ), as shown in Figure 2, which is a function of the total accumulation $n_{i}(k)$

$$
\begin{gathered}
Q_{i, \text { out }}(k)=q_{i, \text { ins }}^{\text {out }}(k)+q_{i, \text { ext }}^{\text {out }}(k)+\sum_{j \in W_{i}} \widehat{m}_{i j}(k)=G_{i}\left(n_{i}(k)\right) \\
G_{i}\left(n_{i}(k)\right)=a_{i} n_{i}^{3}(k)+b_{i} n_{i}^{2}(k)+c_{i} n_{i}(k)
\end{gathered}
$$

where $a_{i}, b_{i}$, and $c_{i}$ are estimated parameters of congested region $i$. The optimal critical accumulation $n_{i, \text { cri }}$ (veh) can be determined based on the fitted MFD curve, i.e., the accumulation value corresponding to the maximum trip completion rate.

Introducing (2) and (3) in (1), we obtain the following dynamic traffic equilibrium equation for the accumulation of region $i$ :

$$
\begin{aligned}
n_{i}(k+1)= & n_{i}(k)+T\left(q_{i, \text { ins }}^{\text {in }}(k)+q_{i, \text { ext }}^{\text {in }}(k)\right. \\
& \left.+\sum_{j \in W_{i}} \widehat{m}_{j i}(k)-G_{i}\left(n_{i}(k)\right)\right) .
\end{aligned}
$$

\section{Coordinated Perimeter Control for Multiple Congested Regions considering Optimized Transfer Flows}

3.1. Multiagent-Based Hierarchical Traffic Management Scheme. Multiagent technology enables several autonomous and intelligent entities, called agents, to have distributed information processing and centralized decision-making capabilities. These agents interact with each other to achieve a desired global goal [31]. The multiagent technology is significantly beneficial to the analysis and resolution of problem domains under the following three conditions: first, the subsystems are geographically distributed; second, these subsystems exist in a dynamic environment; third, subsystems require a high degree of autonomy and flexible interoperability [32]. Inspired by the above, the coordinated perimeter control problem of multiple congested regions is well suited for introducing the concept of multiagent, because of its geographically distributed and dynamic changing nature.

In this section, we first design a multiagent based hierarchical traffic management architecture for two-layer network partitioning including three types of agents: congested region agent (referred to as congested region), global coordination agent, and boundary region agent (referred to as boundary region), as shown in Figure 3 . The first agents are assigned to monitor real-time traffic dynamics of congested regions with different sizes based on MFD theory. The second agent is responsible for receiving communication signals from all the congested region agents about their traffic dynamics and then capturing dynamic equilibrium 


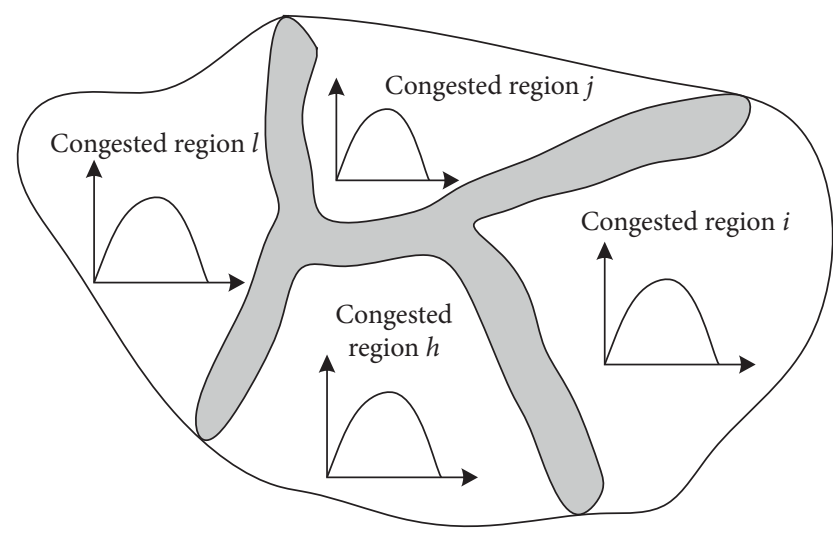

(a)

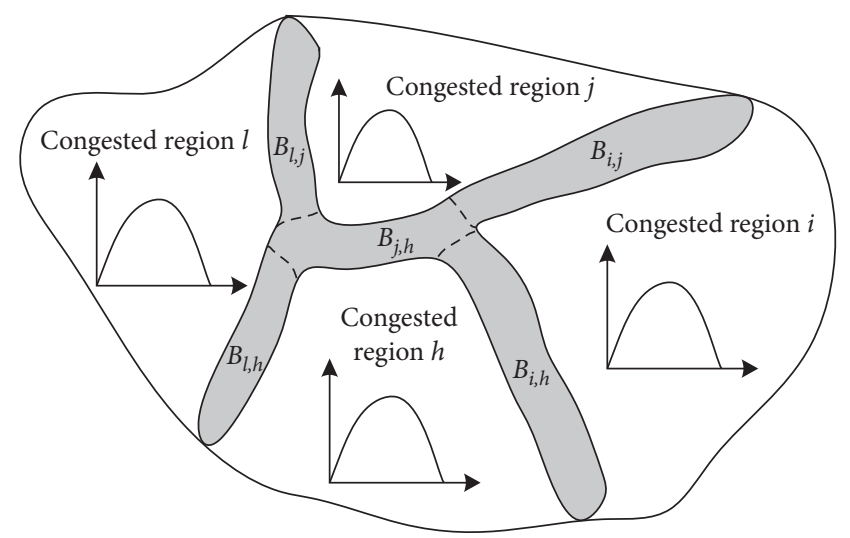

(b)

Figure 1: Illustration of two-layer network partitioning: (a) first-layer division and (b) second-layer division.

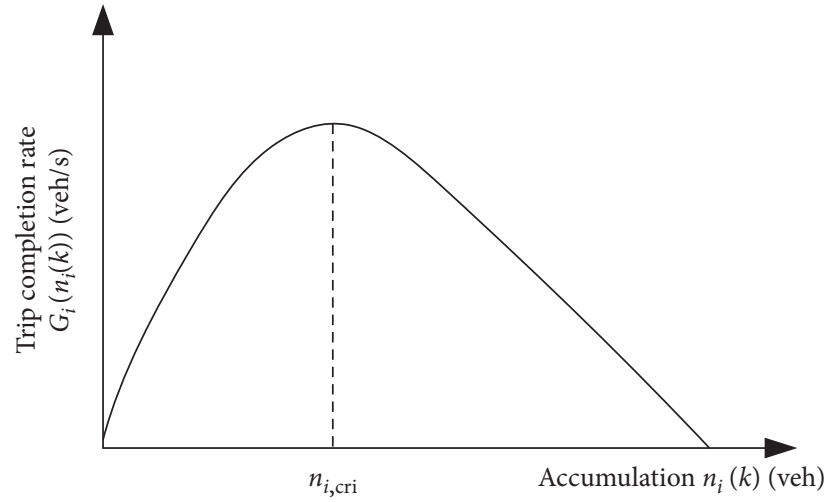

Figure 2: Macroscopic fundamental diagram of congested region $i$.

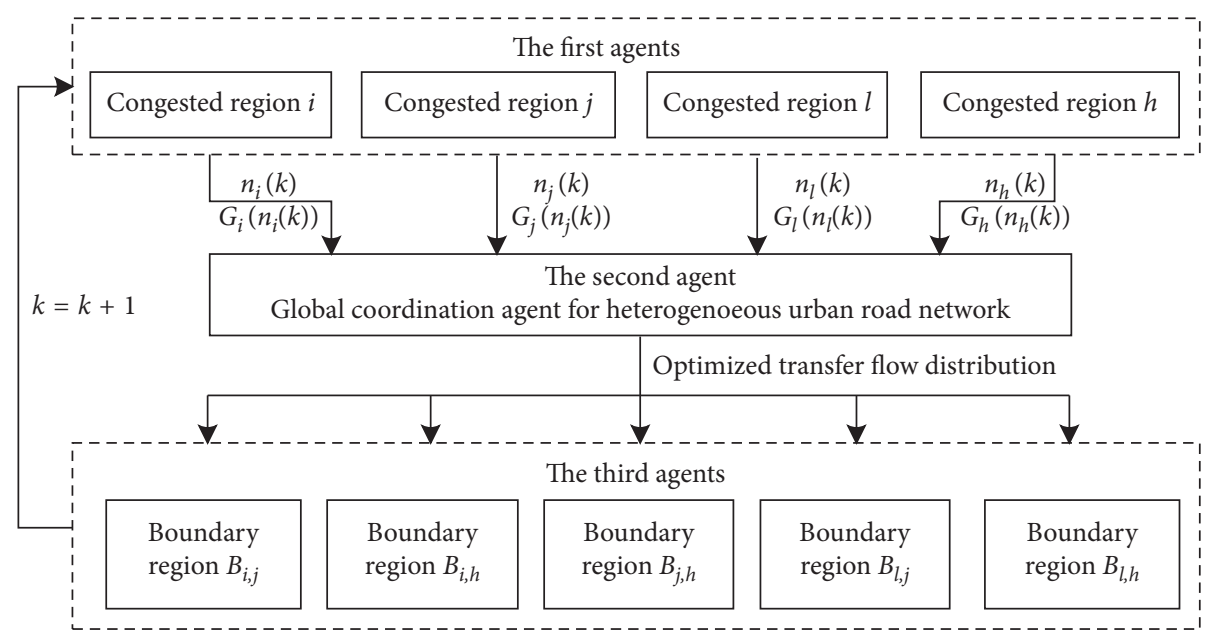

FIGURE 3: Architecture of multiagent-based hierarchical traffic management. 


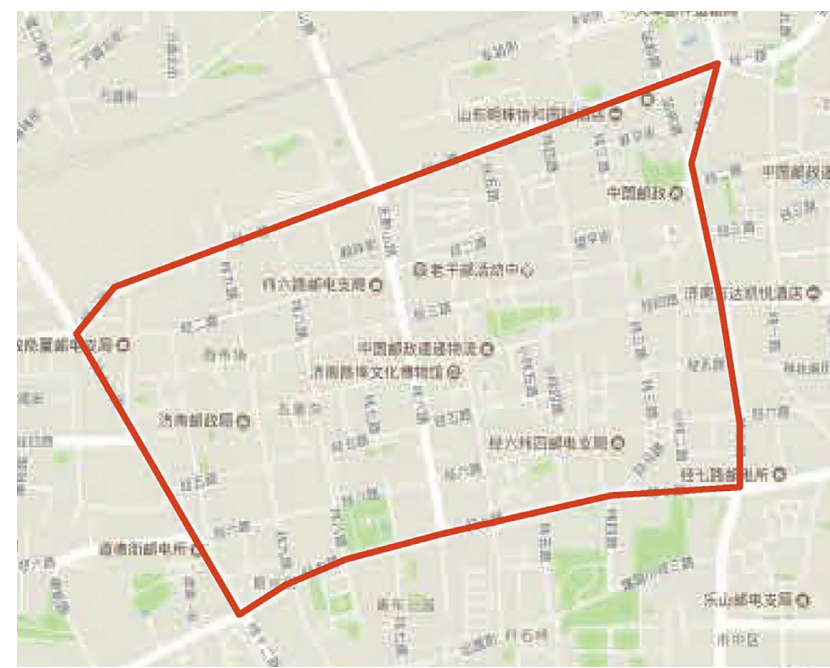

(a)

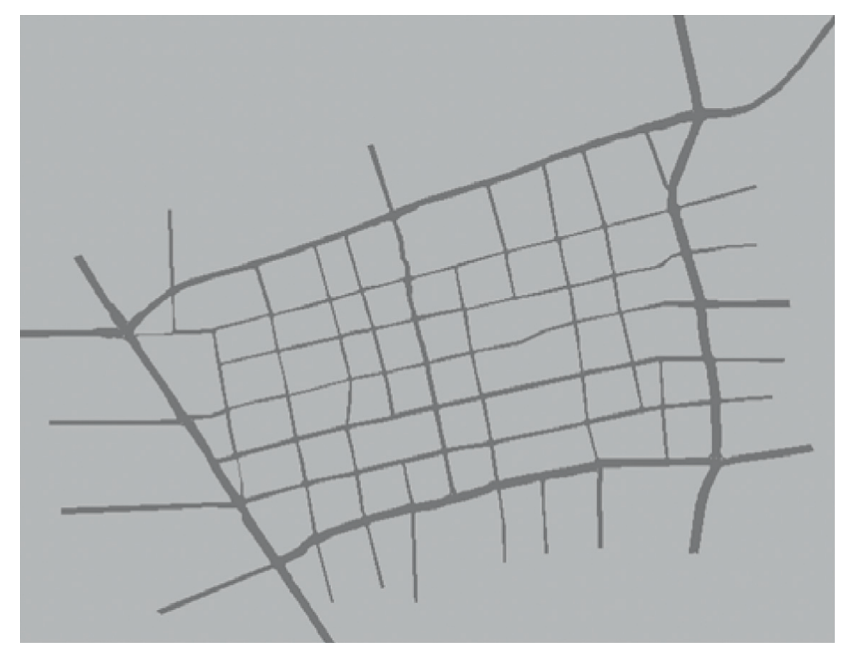

(b)

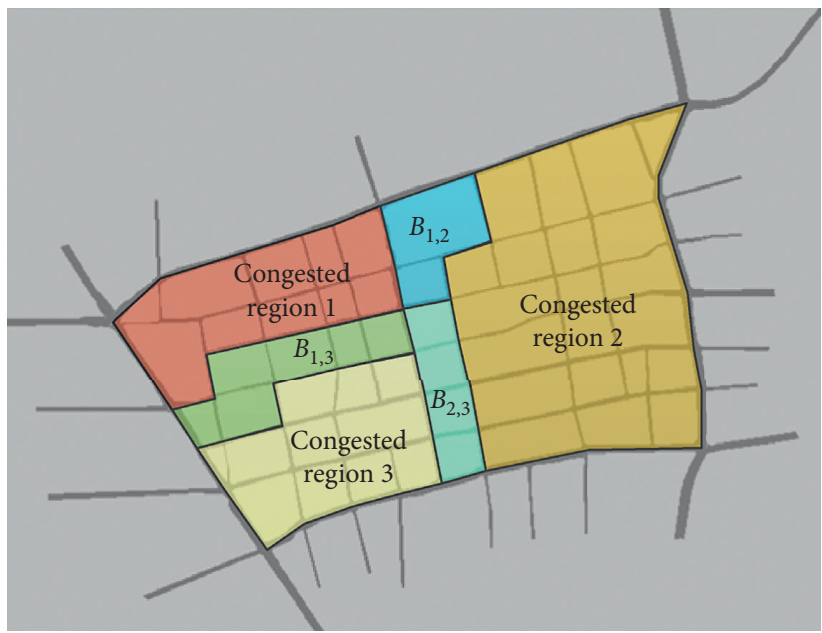

(c)

FIGURE 4: The test site of downtown Jinan: (a) real network, (b) simulation model, and (c) partitioning of the network into 3 congested regions and 3 boundary regions.

conditions of urban network traffic flows. The third agents are designed to perform specific perimeter control task. In the framework of Figure 3, global coordination agent computes the optimal transfer flow distribution of multiple congested regions based on the regional spatial distances and regional traffic states at present time step $k$. Then, global coordination agent outputs the optimized transfer flow distribution to the corresponding boundary regions, which provides the basis for boundary control decisions of each congested region.

\subsection{Transfer Flow Optimization Model of Multiple Congested} Regions. This section is aimed at introducing regional direction choice to find traffic distribution equilibrium conditions for multicongested-region system. Regional direction choice refers to a new decision-making for next neighbouring region made by the vehicles in the currently located congested region. This en-route regional direction decision and the regional route strategies are applied at the aggregated level $[25,33,34]$. Nonetheless, there are some essential differences between these two terms. The basic concept of region-based route is designed to choose a sequence of network regions from a trip's origin region to destination region. If the vehicles travel in accordance with the predefined route, they will not be able to react to the sudden traffic state changes, especially vehicle accidents. The regional direction choice is only a one-step decision for adjacent regions. It can prevent vehicles from traveling to more congested regions in real time. Hence, we assume that the vehicles of congested regions make one-step regional direction decisions, using newly updated traffic information. Based on the above assumption, a transfer flow optimization model is first developed based on an improved multinomial logit (MNL) model, which helps to determine optimal transfer flow distribution and promotes multicongested-region system to gradually achieve network equilibrium. 
The MNL model has been adopted to estimate the region-based direction decisions of vehicles in [33, 35]. And these models are based on travel time of the shortest path from origin region to destination region. If travel time can be measured with a decent level of accuracy, then the logit models developed in these works can properly solve the regional direction decision problem. However, there are large errors in estimating travel time at aggregated level, due to the rough estimation of average travel speed and travel distance. Thus, considering that spatial distribution and traffic state characteristics of multiple congested regions, we improve MNL model by introducing the distances between adjacent congested regions and the realtime traffic states of adjacent congested regions, which aims to obtain optimal transfer flow distribution that balances network traffic flows.

The transfer probability that the vehicles of congested region $i$ head toward the adjacent congested region $j$ at time step $k+1$ is calculated as

$$
\begin{aligned}
p_{i, j}(k+1) & =\frac{\exp \left(-\theta_{\mathrm{d}} R_{d_{i j}}(k)-\theta_{\text {state }} R_{n_{j}}(k)\right)}{\sum_{j \in W_{i}} \exp \left(-\theta_{\mathrm{d}} R_{d_{i j}}(k)-\theta_{\text {state }} R_{n_{j}}(k)\right)}, \\
R_{d_{i j}}(k) & =\frac{d_{i j}(k)}{\left(1 / n_{W_{i}}\right) \sum_{j \in R_{i}} d_{i j}(k)}, \\
R_{n_{j}}(k) & =\frac{n_{j}(k)-n_{j, \mathrm{cri}}}{n_{j, \mathrm{cri}}},
\end{aligned}
$$

where $j$ is the neighbouring congested region of $i ; R_{d_{i j}}(k)$ is the relative distance between congested regions $i$ and $j$ at time step $k$, representing the drivers' perception of spatial distances; $d_{i j}(k)(\mathrm{m})$ is the mean travel distance between congested regions $i$ and $j$ at time step $k ; n_{R_{i}}$ is the number of neighbouring regions that are directly reachable from region $i ; R_{n_{j}}(k)$ is the relative accumulation of congested region $j$ at time step $k$, characterizing the drivers' perception of adjacent regional traffic states; $n_{j}(k)$ is the predicted accumulation of congested region $j$ at time step $k ; n_{j \text {,cri }}$ is the critical accumulation of congested region $j ; \theta_{\mathrm{d}}$ and $\theta_{\text {state }}$ are the influence weight coefficients of the independent variables $R_{d_{i j}}(k)$ and $R_{n_{j}}(k)$.

The value of $R_{n_{j}}(k)$ has the ability of adapting to the changes in real-time accumulation of critical regions. Whether $R_{n_{j}}(k)$ is positive or negative, the transfer probability $p_{i, j}(k)$ will decrease as the value of $R_{n_{j}}(k)$ increases. When the value of $R_{n_{j}}(k)$ is negative, it indicates that there is remaining storage capacity in the adjacent critical region. At this time, the smaller the $R_{n_{j}}(k)$ value is, the larger the number of acceptable vehicles in the adjacent critical region is, and the greater the corresponding transfer probability is.

Since each congested region system evolves slowly with time $k$, we assume that the ratio of the sum of $q_{i, \text { ins }}^{\text {out }}(k)$ and $q_{i, \text { ext }}^{\text {out }}(k)$ to $Q_{i, \text { out }}(k)$ is stable in the process of exploring the optimal transfer flow distribution; i.e., $\rho_{i}=\left(\left(q_{i, \text { ins }}^{\text {out }}(k)+q_{i \text {,ext }}^{\text {out }}(k)\right) /\left(Q_{i \text {,out }}(k)\right)\right), i \in W$. According to transfer flow allocation mechanism as mentioned by Kim et al. [36], the optimized transfer flow from current located congested region $i$ to a neighbor $j$ is determined as follows:

$$
m_{i j}(k+1)=G_{i}\left(n_{i}(k+1)\right) \cdot\left(1-\rho_{i}\right) \cdot p_{i, j}(k+1) .
$$

\subsection{Coordinated Perimeter Control Strategy Based on Model} Predictive Control. Model predictive control (MPC), also known as receding horizon control, is a feedback control strategy. Its working mechanism is that a finite time-domain optimization problem that is composed of objective function and constraints is solved online based on the current state of system and the prediction model at each sampling moment, and the first element of the resulting optimization control sequence is applied to the system. When the system evolves to a new state at the next sampling time, the optimization problem is updated using a new state estimate and the above solution process is repeated. Some of existing studies have adopted MPC approach to solve the perimeter control of large-scale road network [37, 38]. In view of this, we propose a coordinated perimeter control strategy using MPC on the basis of the above transfer flow optimization model, so as to make accumulation of each congested region as close to its optimal critical value $n_{i, \text { cri }}, i \in W$, as possible and maximize their operating efficiency.

The smaller the deviation between real-time accumulations of congested regions and their optimal critical accumulation is, the greater the corresponding trip completion rate and operating efficiency of regional road networks become. Therefore, the optimization objective is to minimize the sum of squares of the ratio of the deviation to the optimal critical accumulation for three congested regions. Then, the perimeter control parameters are introduced as optimization variables to construct a regional-level perimeter signal optimization control problem. The effective transfer flows among multiple congested regions and perimeter inflows from neighbouring external regions are obtained by solving the optimization problem as follows: 
Step 0: determination of the test road network.

Step 1: the first-layer division of network.

(i) Discovering initial congested communities is done according to definition of congestion seed intersection and an intersection with node strength greater than or equal to 3 is chosen as congestion seed intersection.

(ii) Expanding these initial communities is made by adding edges with minimum recursive variance.

(iii) Merging adjacent communities is based on spatial overlap and modularity model. The spatial overlap is the ratio of the number of overlapping links to the number of links after merging. And the calculation of modularity can refer to our recent research [18] for details.

(iv) The congested regions $i, j, h$, and $l$ are extracted.

Step 2: the second-layer division of network.

(i) Based on the extraction results in the previous step, calculate the relative distance between the boundary intersection and the centroid of each congested region. The relative distance $d_{\text {int }, i}$ can be calculated as $d_{\text {int }, i}=\sqrt{\left(\operatorname{lon}_{\text {int }}-\operatorname{lon}_{i}\right)^{2}+\left(\text { lat }_{\text {int }}-\text { lat }_{i}\right)^{2}}$

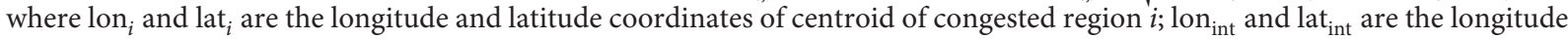
and latitude coordinates of a boundary intersection int.

(ii) Divide the intersections of the gray part in Figure 1(a). The specific basis of subdivision is that a boundary intersection belongs to the boundary region between two congested regions with the smallest sum of relative distances:

(int, $\left.B_{i, j}\right)=\arg \min _{i, j \in W_{\text {int }}}\left(d_{\text {int }, i}+d_{\text {int }, j}\right)$

where $W_{\text {int }}$ is the set of adjacent congested regions of a boundary intersection int; $i$ and $j$ are the congested regions.

(iii) The boundary regions $B_{i, j}, B_{l, j}, B_{j, h}, B_{l, h}$, and $B_{i, h}$ are obtained. Note that the size of boundary region is measured by the number of streets across the boundaries of congested regions, usually up to 2 streets.

Step 3: two-layer network partitioning is derived.

Algorithm 1: Two-layer partitioning algorithm of heterogeneous urban networks.

$$
\begin{array}{ll}
\min _{\mu_{i, \mathrm{ext}}, \mu_{i j}} \quad J=\sum_{i \in W} \sum_{l=0}^{N_{l}-1}\left(\frac{n_{i}\left(k_{c}+l\right)-n_{i, \mathrm{cri}}}{n_{i, \mathrm{cri}}}\right)^{2} \\
\text { s.t. } \quad \text { for } l=0, \ldots, N_{l}-1: \\
\\
n_{i}\left(k_{c}+l+1\right)=n_{i}\left(k_{c}+l\right)+T_{c}\left(q_{i, \text { ins }}^{\text {in }}\left(k_{c}+l\right)+\mu_{i, \mathrm{ext}}\left(k_{c}+l\right) q_{i, \mathrm{ext}}^{\text {in }}\left(k_{c}+l\right)+\sum_{j \in W_{i}} \widehat{m}_{j i}\left(k_{c}+l\right)-G_{i}\left(n_{i}\left(k_{c}+l\right)\right)\right) \\
& \widehat{m}_{i j}\left(k_{c}+l\right)=\mu_{i j}\left(k_{c}+l\right) m_{i j}\left(k_{c}+l\right) \\
& \mu_{\min } \leq \mu_{i, \mathrm{ext}}\left(k_{c}+l\right) \leq \mu_{\max } \\
& \mu_{\min } \leq \mu_{i j}\left(k_{c}+l\right) \leq \mu_{\max } \\
& 0 \leq \widehat{m}_{i j}\left(k_{c}+l\right) \leq C_{i j} \\
& \text { if } 1 \leq l \leq N_{l}-1 \\
& q_{i, \mathrm{ext}}^{\text {in }}\left(k_{c}+l\right)=q_{i, \mathrm{ext}}^{\text {in }}\left(k_{c}\right) \\
& \mu_{i j}\left(k_{c}+l\right)=\mu_{i j}\left(k_{c}\right),
\end{array}
$$

where $k_{c}$ is the sequence of perimeter control cycle $T_{c} ; N_{l}$ is the prediction horizon; $\mu_{i \text { ext }}\left(k_{c}+l\right)$ is the perimeter control variable of inflows from neighbouring external region to congested region $i$, which is the proportion of the external inflows that are allowed to enter congested region $i ; \mu_{i j}\left(k_{c}+\right.$ $l)$ is the perimeter control variable of transfer flows from congestion region $i$ to $j ; \widehat{m}_{i j}\left(k_{c}+l\right)$ is the effective transfer flow that is the product of the optimization variable $\mu_{i j}\left(k_{c}+\right.$ $l)$ and the optimized transfer flow $m_{i j}\left(k_{c}+l\right)(\mathrm{veh} / \mathrm{s}) ; \mu_{\min }$ and $\mu_{\max }$ are the minimum and maximum values of perimeter control variables, respectively; $C_{i j}(\mathrm{veh} / \mathrm{s})$ is maximum traffic flow from congested region $i$ to $j$. In addition, it should be noted that the perimeter control variables are equal to the first perimeter control cycle when $1 \leq l \leq N_{l}-1$. 

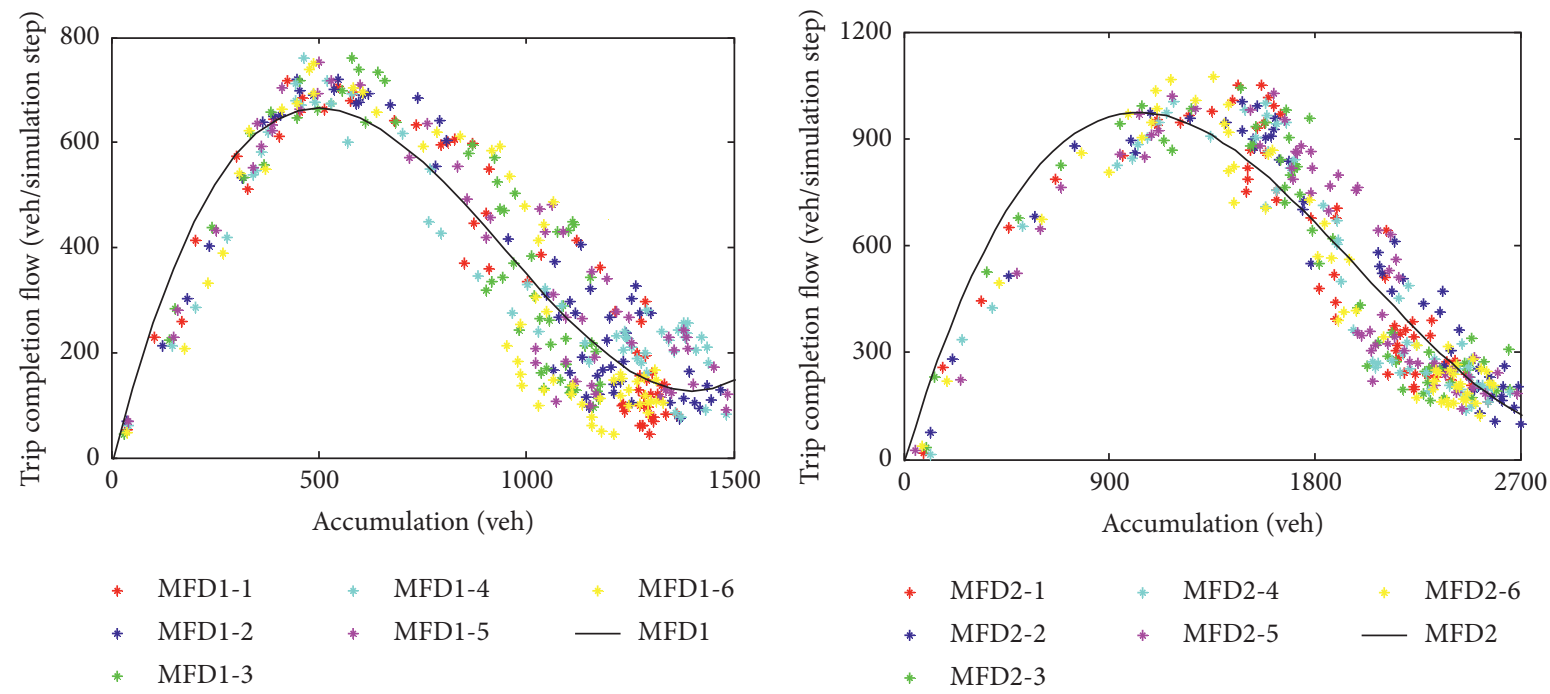

(a)

(b)

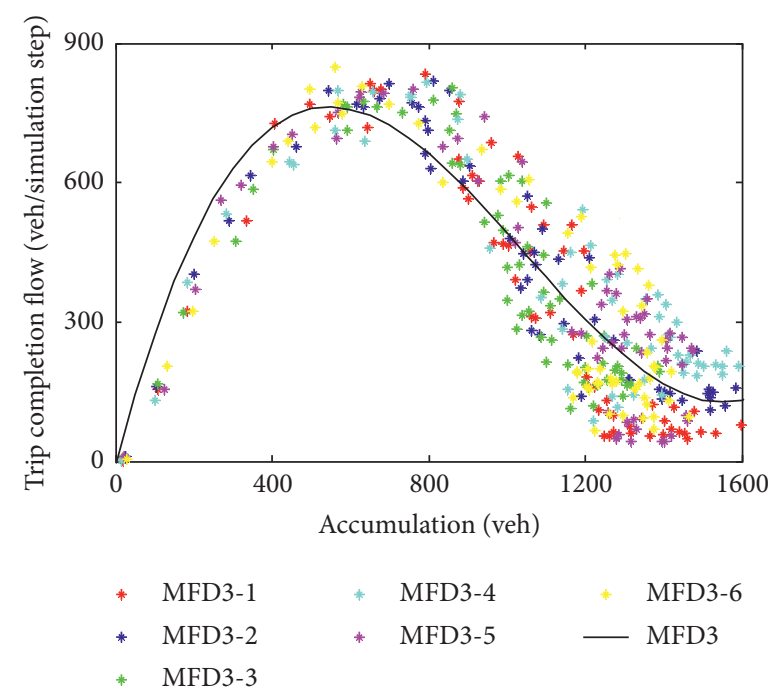

(c)

FIGURE 5: Macroscopic fundamental diagrams for (a) congested region 1, (b) congested region 2, and (c) congested region 3.

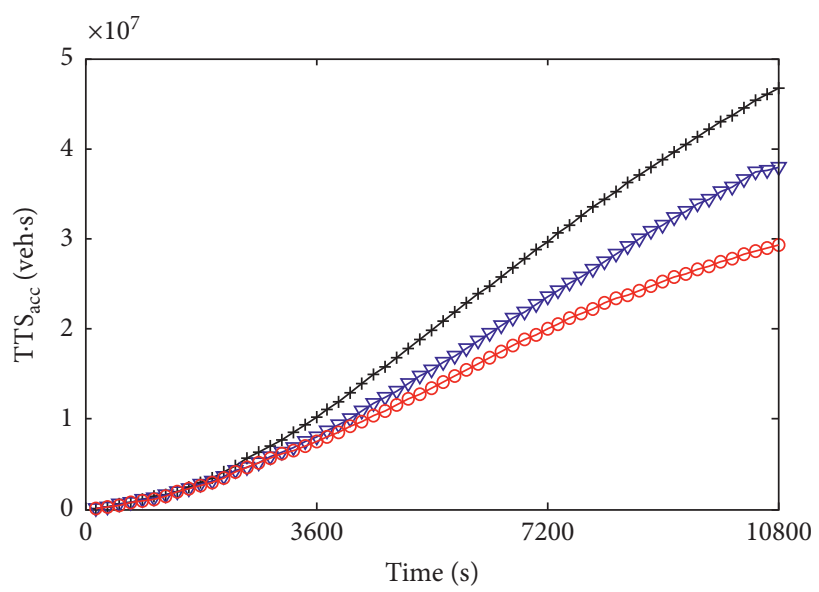

$$
\begin{aligned}
& + \text { Fixed signal control } \\
& -\quad \text { Decentralized control } \\
& -\quad \text { The proposed coordinated control }
\end{aligned}
$$

FIgURE 6: Time series of TTS $_{\text {acc }}$ in the experimental area.

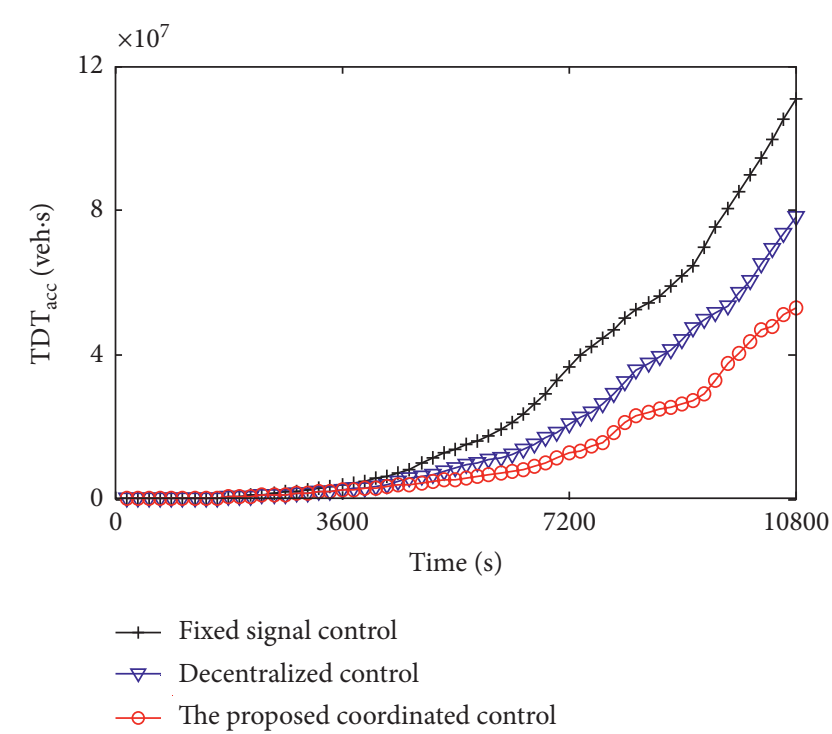

FIgURE 7: Time series of $\mathrm{TDT}_{\mathrm{acc}}$ in the experimental area. 
TABle 1: Traffic inflows of all the border links in the experimental area.

\begin{tabular}{lccccccr}
\hline \multirow{2}{*}{ Traffic demand } & \multicolumn{5}{c}{ Simulation time (s) } \\
& $0-900$ & $900-1800$ & $1800-2700$ & $2700-4500$ & $4500-6300$ & $6300-8100$ & $8100-10800$ \\
\hline Traffic inflows (veh/h/lane) & 600 & 800 & 1000 & 1200 & 900 & 600 & 200 \\
\hline
\end{tabular}

To better achieve the effective transfer flow distribution of multiple congested regions obtained by the above model, we adopt the following formula to convert the effective transfer flows into the signal control parameters of boundary intersections; namely,

$$
g_{i j, h}(k)=\widehat{m}_{i j}\left(k_{c}\right) \cdot \frac{T}{T_{c}} \cdot \frac{C_{i j, h}}{C_{i j}} \cdot \frac{g_{i j, h}(k-1)}{q_{i j, h}(k-1)}
$$

where $g_{i j, h}(k)(s)$ is the phase green duration of traffic flow on border link $h$ from congested region $i$ to $j$ at time step $k$; T (s) is the simulation step; $C_{i j, h}(\mathrm{veh} / \mathrm{s})$ is the maximum traffic flow of border link $h$ from congested region $i$ to $j$, that is, the product of the lane number of border links $h$ and the saturation flow rate; $q_{i j, h}(k-1)(\mathrm{veh} / T)$ is the measured traffic flow of border link $h$ from congested region $i$ to $j$ at time step $k-1$.

\section{Case Study and Results}

4.1. Network Description and Simulation Setup. To verify the effectiveness of the proposed perimeter control strategy in this paper, a part of road network in downtown Jinan is selected as the test site, including about 74 intersections and 147 links with lengths varying from 64 to 476 meters. The representation of the experimental area on the map can be shown in Figure 4(a). Figure 4(b) presents the simulation scene of road network based on VISSIM. Using the two-layer partitioning of heterogeneous urban networks, the experimental area is divided into 3 homogeneous congested regions and 3 boundary regions. The specific partition results are shown in Figure 4(c).

In this simulation environment, all signalized intersections operate with two-phase fixed signal timing plans on a common cycle length of $90 \mathrm{~s}$. Data detection point is placed in the middle of each link. And each link is provided with a delay detection section. The free flow speed of vehicles on links is $45-50 \mathrm{~km}$ per hour. Traffic demand is realized by the incoming flows of all the border links in the experimental area. The specific inflows are shown in Table 1. During this demand scenario, the inflows of the experimental network gradually increase at the beginning and then decrease, which simulates the evolution of regional traffic flows experiencing a congestion peak.

4.2. MFD Models of Multiple Congested Regions. To derive the accurate MFD models of multiple congested regions, a simulation experiment was first carried out without using any perimeter control strategy. All the intersections in test area adopt well-tuned fixed signal timing plans, and the traffic demand scenario is implemented according to traffic inflows of all the border links in Table 1. The simulation duration is set to $3 \mathrm{~h}$ and the simulation step is $180 \mathrm{~s}$. Considering the stochastic nature of the microscopic simulator, this simulation experiment with the same traffic demand was repeated 6 times. As shown in Figure 5, six groups of discrete points with different colors correspond to repeated experiments with different random seeds, denoted by MFD $i-n, n \in\{1,2,3,4,5,6\}$ and $i \in\{1,2,3\}$. Each discrete point represents the accumulation and corresponding trip completion flow of each congested region within $180 \mathrm{~s}$. According to the data of all discrete points in each congested region, their MFD curves were obtained using the curve fitting function in MATLAB, that is, the solid black curve in Figure 5. The MFD curve fitting parameters of three congested regions are $a_{1}=1.4619 \times 10^{-6}, b_{1}=-0.0041629, c_{1}=$ $3.0567, a_{2}=2.3401 \times 10^{-7}, b_{2}=-0.0014146, c_{2}=2.1631$, $a_{3}=1.2540 \times 10^{-6}, \quad b_{3}=-0.0039474, \quad$ and $\quad c_{3}=3.1924$. Furthermore, the peak value of the MFD curve is used to determine the optimal critical accumulation of each congested region; namely, $n_{1, \text { cri }}=500 \mathrm{veh}, n_{2, \text { cri }}=1050 \mathrm{veh}$, and $n_{3, \text { cri }}=550$ veh.

4.3. Results and Analysis of Coordinated Perimeter Control. In this section, much more attention is paid to verify the feasibility and effectiveness of coordinated perimeter control strategy proposed in this paper. Traffic demand of this simulation experiment is consistent with the MFD model acquisition experiment of multiple congested regions, as shown in Table 1 . The simulation duration is set to $3 \mathrm{~h}$, and the last $0.5 \mathrm{~h}$ is used to clear traffic flows of experiment network. The simulation step and perimeter control cycle $T_{c}$ are $180 \mathrm{~s}$

Three different control strategies are designed. First of all, we regard fixed signal control as the base case. The simulation network operates with a set of well-tuned fixed signal timing plans and there is no restriction on perimeter traffic flows. The performance of different perimeter control strategies can be evaluated on the basis of the improvements that they can achieve compared to the base case. Then, one option is that the discrete boundary-feedback-control strategy [39] is adopted to adjust signal timing plans of all the boundary intersections for three congested regions, and the signal control of intersections within the experiment area remains the same as the fixed signal control scenario. The perimeter control of three congested regions is independent of each other, referred to as decentralized control. Another alternative is to apply the proposed coordinated perimeter control strategy based on optimized transfer flows in this paper (referred to as the proposed coordinated control), combined with the fixed signal control within the experiment area. It should be noted that the decentralized control and the proposed coordinated control adjust the signal timing plans of the same boundary intersections, including 

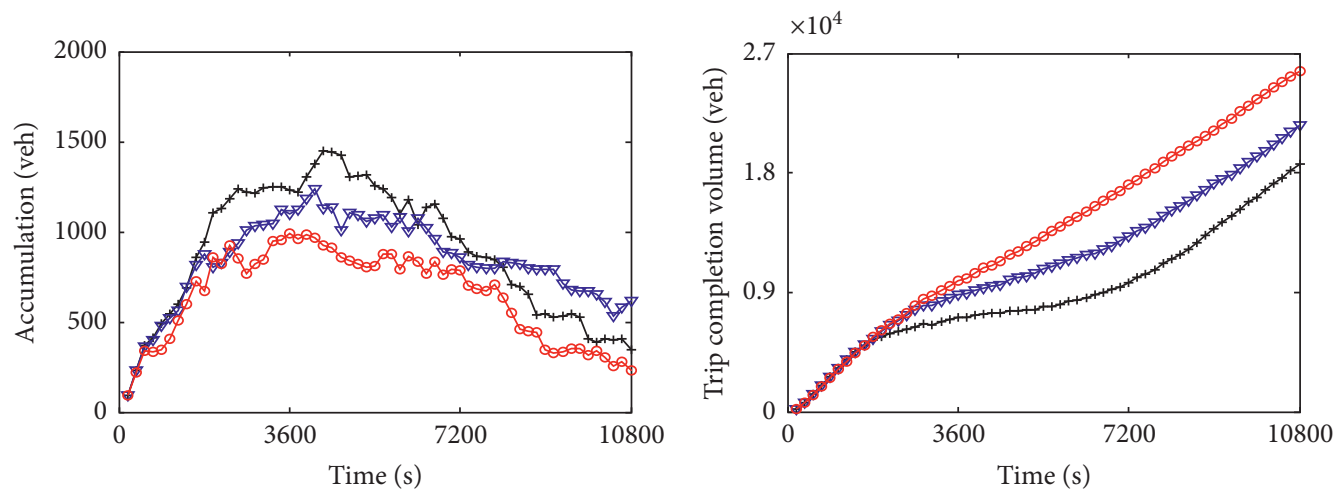

+. Fixed signal control

$\rightarrow$ Decentralized control

$\rightarrow$ The proposed coordinated control

(a)

\section{- Fixed signal control \\ $\rightarrow$ Decentralized control \\ $\rightarrow$ The proposed coordinated control}

(b)

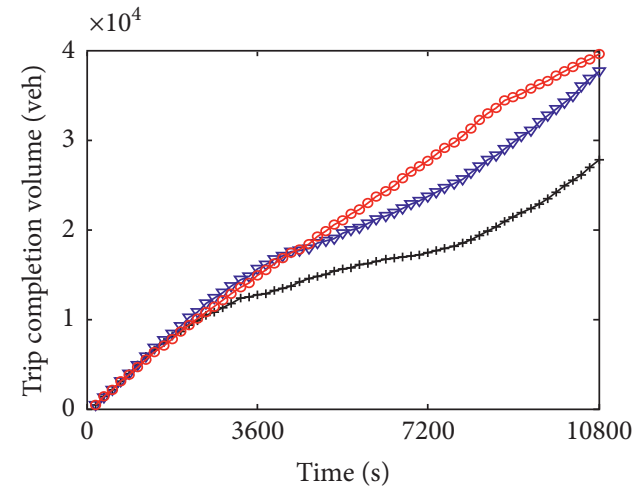

$\longrightarrow$ Fixed signal control

$\rightarrow$ Decentralized control

$\rightarrow$ The proposed coordinated control

(d)

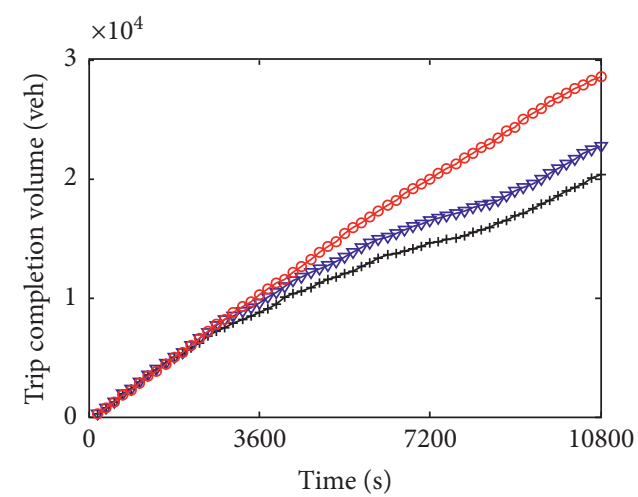

- Fixed signal control

$\rightarrow$ Decentralized control

- The proposed coordinated control

(f)

Figure 8: Accumulation and trip completion volume of three congested regions. (a) Accumulation of congested region 1. (b) Trip completion volume of congested region 1. (c) Accumulation of congested region 2. (d) Trip completion volume of congested region 2. (e) Accumulation of congested region 3. (f) Trip completion volume of congested region 3. 

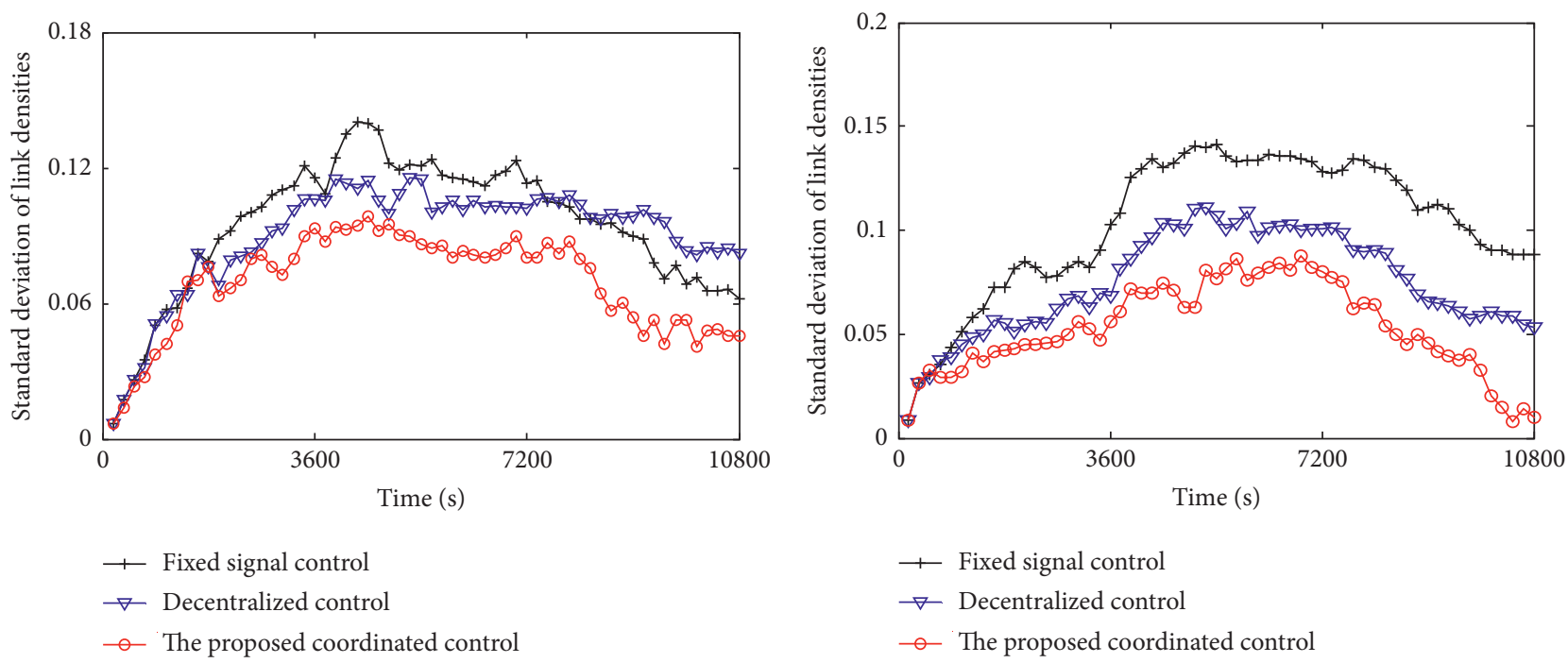

(b)

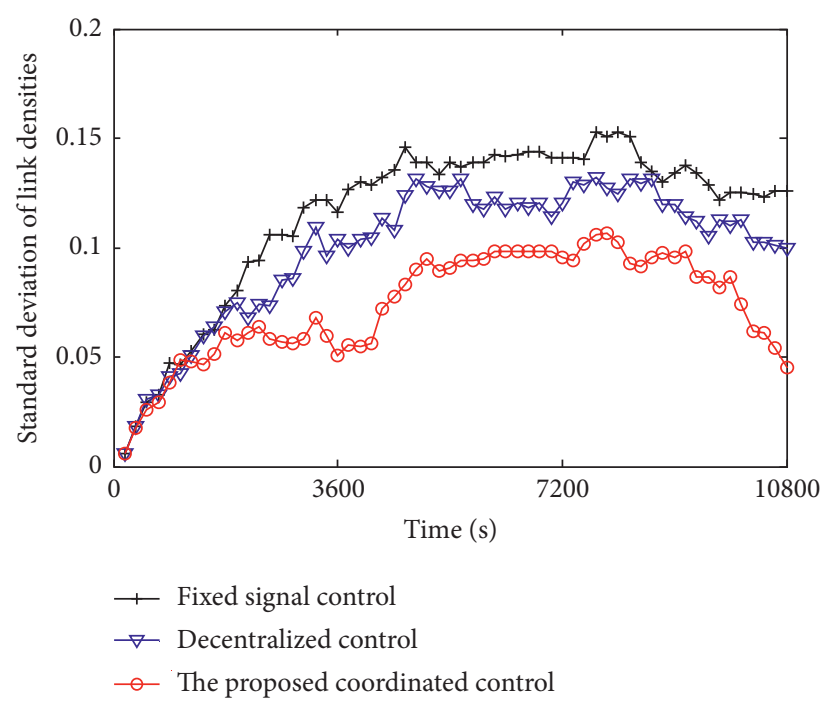

(c)

FIgURE 9: Standard deviation of link densities for (a) congested region 1, (b) congested region 2, and (c) congested region 3.

TABLE 2: $\mathrm{TTS}_{\mathrm{acc}}$ and $\mathrm{TDT}_{\mathrm{acc}}$ of three simulation control strategies.

\begin{tabular}{lccccc}
\hline Performance indicators & $\begin{array}{c}\text { Fixed signal } \\
\text { control }\end{array}$ & $\begin{array}{c}\text { Decentralized } \\
\text { control }\end{array}$ & Improvement (\%) & $\begin{array}{c}\text { The proposed } \\
\text { coordinated control }\end{array}$ & Improvement (\%) \\
\hline $\operatorname{TTS}_{\text {acc }}\left(\times 10^{7} \mathrm{veh} \cdot \mathrm{s}\right)$ & 4.68 & 3.80 & 18.8 & 2.94 & 37.2 \\
$\operatorname{TDT}_{\mathrm{acc}}\left(\times 10^{7} \mathrm{veh} \cdot \mathrm{s}\right)$ & 11.07 & 7.81 & 29.4 & 5.30 & 52.1 \\
\hline
\end{tabular}

the signalized intersections of three boundary regions and the remaining boundary intersections of congested regions.

In the simulation experiment of the proposed coordinated control in this paper, the minimum and maximum values of perimeter control variables are chosen as $\mu_{\text {min }}=0.1$ and $\mu_{\max }=0.9$. The prediction horizon is selected as $N_{l}=4$ for the MPC scheme. The saturation flow rate of border links is $1800 \mathrm{veh} / \mathrm{h} /$ lane, and the minimum green duration is $10 \mathrm{~s}$. Assuming that the range of congested regions is constant over the simulation, the parameters of transfer flow optimization model are set as $\theta_{\mathrm{d}}=0.55, \theta_{\text {state }}=0.45$, $R_{d_{12}}(k)=1.33, \quad R_{d_{13}}(k)=0.67, \quad R_{d_{21}}(k)=1.04$, $R_{d_{23}}(k)=0.96, \quad R_{d_{31}}(k)=0.71$, and $R_{d_{32}}(k)=1.29$. The proposed coordinated control takes about $2.38 \mathrm{~s}$ at each simulation step, which is smaller than the sampling time. This shows that the proposed method in this paper has realtime feasibility.

Two evaluation indicators for the entire experimental area are introduced to compare traffic operating performance of three simulation control strategies. The first one is 
accumulated amount of total time spent TTS $_{\text {acc }}$ by all vehicles in the experimental area. The second one is accumulated total delay time $\mathrm{TDT}_{\mathrm{acc}}$ of all vehicles in the experimental area. The specific calculation formulas are as follows:

$$
\mathrm{TTS}_{\mathrm{acc}}=\sum_{k_{c}=1}^{K_{c}} T_{c} \cdot n\left(k_{c}\right), \mathrm{TDT}_{\mathrm{acc}}=\sum_{k_{c}=1}^{K_{c}} \bar{t}_{d}\left(k_{c}\right) \cdot n\left(k_{c}\right),
$$

where $n\left(k_{c}\right)$ (veh) is the total accumulation in the experimental area at perimeter control cycle $k_{c} ; \bar{t}_{d}\left(k_{c}\right)(\mathrm{s})$ is the average delay of each vehicle in the experimental area at perimeter control cycle $k_{c}$, which can be directly obtained by VISSIM; $K_{c}$ is the number of perimeter control cycles. Figures 6 and 7 display the time series of two performance indicators in the experimental area under three simulation control strategies, respectively. Table 2 gives the comparative analysis of the corresponding network operating performance.

As illustrated in Figure 6, the proposed coordinated control and the decentralized control produce significantly decreased values of $\mathrm{TTS}_{\mathrm{acc}}$, compared to the fixed signal control. During the first 10 perimeter control cycles, since the traffic of road network is not congested, the difference among three simulation control strategies is not obvious. With the continuous increase of traffic demand, the proposed coordinated control in this paper gradually shows better performance than the decentralized control and the fixed signal control. At the end of the simulation, the improvements of the proposed coordinated control and the decentralized control in terms of TTS $_{\text {acc }}$ over the fixed signal control are $37.2 \%$ and $18.8 \%$, respectively, as shown in Table 2. From Figure 7 , the proposed coordinated control and the decentralized control could obviously reduce $\mathrm{TDT}_{\mathrm{acc}}$ compared with the fixed signal control. In the first 25 perimeter control cycles, the difference for $\mathrm{TDT}_{\mathrm{acc}}$ of two perimeter control strategies is small. Subsequently, the gap for $\mathrm{TDT}_{\mathrm{acc}}$ between the proposed coordinated control and the decentralized control is gradually widened, and the main reason is that the proposed coordinated control in this paper reduces the vehicle delays of boundary regions. At the end of the entire simulation experiment, the proposed coordinated control and the decentralized control reduced 52.1\% and $29.4 \%$ of TDT $_{\text {acc }}$ over the fixed signal control, respectively, as shown in Table 2. For traffic performance of the entire experimental area, the proposed coordinated control strategy is superior to the decentralized control strategy and the fixed signal control strategy.

Three evaluation indicators for each congested region are used to compare traffic operating performance of three simulation control strategies, such as accumulation, trip completion volume, and standard deviation of link densities. The standard deviation SD of link densities could be calculated by the following formula:

$$
\mathrm{SD}_{i}\left(k_{c}\right)=\sqrt{\frac{1}{V_{i}} \sum_{v \in L_{i}}\left(\frac{n_{i, v}\left(k_{c}\right)}{\lambda_{i, v} l_{i, v}}-\bar{d}_{i}\left(k_{c}\right)\right)^{2}, \bar{d}_{i}\left(k_{c}\right)=\frac{1}{V_{i}} \sum_{v \in L_{i}} \frac{n_{i, v}\left(k_{c}\right)}{\lambda_{i, v} l_{i, v}}},
$$

where $\mathrm{SD}_{i}\left(k_{c}\right)$ is the standard deviation of link densities for congested region $i$ at perimeter control cycle $k_{c} ; n_{i, v}\left(k_{c}\right)$ (veh) is the accumulation of link $v$ in congested region $i$ at perimeter control cycle $k_{c} ; \lambda_{i, v}$ and $l_{i, v}(\mathrm{~m})$ are the number of lanes and the length for link $v$ in congested region $i$, respectively; $L_{i}$ is the set of links in congested region $i ; V_{i}$ is the number of links in congested region $i ; \bar{d}_{i}\left(k_{c}\right)$ is the average value of link densities in congested region $i$ at perimeter control cycle $k_{c}$. Figures 8 and 9 display traffic operating performance of three congested regions under different simulation control strategies.

As can be observed from Figures $8(\mathrm{c})$ and $8(\mathrm{e})$, the proposed coordinated control maintains real-time accumulations of congested regions 2 and 3 around their optimal critical values at the beginning of the simulation. As traffic demand increases, the accumulations of these two congested regions grow to a certain extent with the proposed coordinated control, but they are significantly better than those of the decentralized control and the fixed signal control. For the last hour of the simulation, their accumulations with the proposed coordinated control begin to fade at a rate that is faster than that of the other two control strategies. As illustrated in Figure 8(a), the proposed coordinated control could make the accumulation of congested region 1 closer to its optimal critical value compared to the decentralized control and the fixed signal control, but its overall accumulation state is not as good as that of other two congested regions. This is mainly due to two reasons: (i) the number of gated boundary intersections for congested region 1 is less than that of the other two congested regions; (ii) the proposed coordinated control strategy makes a compromise when cooperatively regulating the distribution of regional accumulation, that is, sacrificing the partial accumulation state of congested region 1 , so as to ensure that the overall operating performance of three congested regions is optimal. As can be seen from Figures (8b), 8(d) and $8(\mathrm{f})$, at the early stage of simulation experiment, three simulation control strategies generate similar trip completion volumes for congested regions 1 or 3 . As regional traffic congestion gets heavier, the proposed coordinated control strategy achieves larger trip completion volumes for congested regions 1 and 3 than the other two strategies. The initial trip completion volume of congested region 2 with the proposed coordinated control is smaller than that with decentralized control, due to the fact that the corresponding accumulation of congested region 2 is relatively lower (see Figure 8(c)). Then, the proposed coordinated control enables a higher trip completion volume of congested region 2 than the other two comparison strategies. On the whole, the fixed signal control lacks the optimal control of network traffic flows, which causes the oversaturation of three congested regions and 
even gridlocks of road network; the decentralized control does not consider the interaction and coordination among congested regions, and it could only better improve the operating states of individual congested regions and ignore traffic performance of the entire experimental area; the proposed coordinated control strategy in this paper not only could evenly distribute the accumulations of multiple congested regions, but also ensure the improvement of operation efficiency for the entire experimental area.

As can be observed from Figures 9(a) and 9(c), three simulation control strategies in terms of the SD have almost the same change pattern for congested regions 1 or 3 at the beginning and then exhibit the clear differences after about 1800 s. Compared to the fixed signal control, both the proposed coordinated control and the decentralized control reduce the $\mathrm{SD}$ of congested regions 1 and 3, and the improvements of the coordinated control are significantly greater. The results reveal that our proposed coordinated control outperforms the decentralized control in balancing spatial distribution of regional traffic flows. As depicted in Figure 9(b), the SD of congested region 2 under the coordinated control strategy is lower than 0.05 at the beginning, which lasts for nearly 1 hour. After experiencing a certain degree of SD growth for congested region 2, the homogeneity of road network gradually increases again with the decrease of traffic demand. For congested region 2, the proposed coordinated control strategy produces better results than the other two control strategies. From the perspective of the entire experimental area, the coordinated control strategy in this paper could simultaneously balance traffic flow distribution of three congested regions, which achieves increased homogeneity and maximum utilization of network capacity in each congested region.

\section{Conclusion and Future Work}

This paper first extends our previous research [18] to present a two-layer network partitioning method that can accurately capture spatially traffic distribution characteristics of heterogeneous urban networks with multiple centers of congestion. Based on this partition, traffic flow equilibrium model using MFD is introduced to characterize traffic dynamics of multiregion system. Then, a multiagent based hierarchical traffic management scheme is proposed. In this scheme, we develop a coordinated perimeter control strategy based on optimized transfer flows, which is aimed at enabling each individual congested region to track their desired accumulations as much as possible and achieving dynamic equilibrium of traffic flows in road networks. The main contributions of this research focus on the following aspects: (a) it could effectively tackle traffic optimization control problem in multiple congested regions that are not directly connected in an urban network; (b) it could achieve traffic dynamic equilibrium of an urban network with multiple congested regions by coordinated perimeter control strategy considering optimized transfer flows; (c) it explores the application effect of heterogeneous network partitioning and coordinated perimeter control in real road networks.
For future research, the effect of different boundary region sizes on the coordinated perimeter control strategy should be explored. And when the size of boundary region is larger, the corresponding traffic signal coordinated control method needs to be further studied. Additionally, based on the existing traffic data (e.g., fixed detection data and GPSbased floating car data), real-time accurate estimation method of MFD curve and critical accumulation should also be research priorities. This could be implemented using big data analysis or machine learning techniques.

\section{Data Availability}

The data used to support the findings of this study are available from the corresponding author upon request.

\section{Conflicts of Interest}

The authors declare that there are no conflicts of interest regarding the publication of this paper.

\section{Acknowledgments}

This work was supported by the National Natural Science Foundation of China (Grant no. ZR2017MF011), Shandong Natural Science Foundation of China (Grant no. ZR2018MF027), Shandong Key Research and Development Project of China (Grant no. GG201809240117), Jinan Science and Technology Bureau Project (Grant no. 2019GXRC022), Intelligent System and Equipment (Advanced Vehicle, Robot System) Innovation Team (Grant no. SDJTUC180), and Jinan Philosophy and Social Science Planning Program (Grant no. JNSK18D15).

\section{References}

[1] J. Y. K. Luk, "Two traffic-responsive area traffic control methods: SCAT and SCOOT," Traffic Engineering and Control, vol. 25, no. 1, pp. 14-22, 1984.

[2] P. B. Hunt, D. I. Robertson, R. D. Bretherton, and M. C. Royle, "The SCOOT on-line traffic signal optimization technique," Traffic Engineering and Control, vol. 23, no. 4, pp. 190-192, 1982.

[3] M. Keyvan-Ekbatani, M. Papageorgiou, and I. Papamichail, "Urban congestion gating control based on reduced operational network fundamental diagrams," Transportation Research Part C: Emerging Technologies, vol. 33, pp. 74-87, 2013.

[4] A. Aalipour, H. Kebriaei, and M. Ramezani, "Analytical optimal solution of perimeter traffic flow control based on MFD dynamics: a Pontryagin's maximum principle approach," IEEE Transactions on Intelligent Transportation Systems, vol. 20, no. 9, pp. 3224-3234, 2019.

[5] K. Yang, N. Zheng, and M. Menendez, "Multi-scale perimeter control approach in a connected-vehicle environment," Transportation Research Part C: Emerging Technologies, vol. 94, pp. 32-49, 2018.

[6] M. Amirgholy and H. O. Gao, "Modeling the dynamics of congestion in large urban networks using the macroscopic fundamental diagram: user equilibrium, system optimum, and pricing strategies," Transportation Research Part B: Methodological, vol. 104, pp. 215-237, 2017. 
[7] Z. Gu, S. Shafiei, Z. Liu, and M. Saberi, "Optimal distance- and time-dependent area-based pricing with the network fundamental diagram," Transportation Research Part C: Emerging Technologies, vol. 95, pp. 1-28, 2018.

[8] D. Wei and Z. Yang, "Bi-level route guidance method for large-scale urban road networks," EURASIP Journal on Wireless Communications and Networking, vol. 2019, no. 1, p. 127, 2019.

[9] M. Liu, D. Han, D. Li, and M. Wang, "Route guidance during evacuations integrated with perimeter control strategy in large-scale mixed traffic flow networks," International Journal of Modern Physics C (IJMPC), vol. 29, no. 11, pp. 1-19, 2018.

[10] Y. Han, M. Ramezani, A. Hegyi, Y. Yuan, and S. Hoogendoorn, "Hierarchical ramp metering in freeways: an aggregated modeling and control approach," Transportation Research Part C: Emerging Technologies, vol. 110, pp. 1-19, 2020.

[11] M. Yildirimoglu and M. Ramezani, "Demand management with limited cooperation among travellers: a doubly dynamic approach," Transportation Research Part B: Methodological, vol. 132, pp. 267-284, 2020.

[12] C. Buisson and C. Ladier, "Exploring the impact of homogeneity of traffic measurements on the existence of macroscopic fundamental diagrams," Transportation Research Record: Journal of the Transportation Research Board, vol. 2124, no. 1, pp. 127-136, 2009.

[13] A. Zockaie, M. Saberi, and R. Saedi, "A resource allocation problem to estimate network fundamental diagram in heterogeneous networks: optimal locating of fixed measurement points and sampling of probe trajectories," Transportation Research Part C: Emerging Technologies, vol. 86, pp. 245-262, 2018.

[14] V. L. Knoop, H. Van Lint, and S. P. Hoogendoorn, "Traffic dynamics: its impact on the macroscopic fundamental diagram," Physica A: Statistical Mechanics and Its Applications, vol. 438, pp. 236-250, 2015.

[15] M. Saeedmanesh and N. Geroliminis, "Clustering of heterogeneous networks with directional flows based on "Snake" similarities," Transportation Research Part B: Methodological, vol. 91, pp. 250-269, 2016.

[16] M. Saeedmanesh and N. Geroliminis, "Dynamic clustering and propagation of congestion in heterogeneously congested urban traffic networks," Transportation Research Part B: Methodological, vol. 105, pp. 193-211, 2017.

[17] Z. Zhou, S. Lin, and Y. Xi, "A dynamic network partition method for heterogenous urban traffic networks," in Proceedings of the 15th International IEEE Conference on Intelligent Transportation Systems, pp. 820-825, IEEE, Anchorage, AK, USA, September 2012.

[18] Y. Guo, L. Yang, S. Hao, and J. Gao, "Dynamic identification of urban traffic congestion warning communities in heterogeneous networks," Physica A: Statistical Mechanics and Its Applications, vol. 522, pp. 98-111, 2019.

[19] M. Keyvan-Ekbatani, A. Kouvelas, I. Papamichail, and M. Papageorgiou, "Exploiting the fundamental diagram of urban networks for feedback-based gating," Transportation Research Part B: Methodological, vol. 46, no. 10, pp. 13931403, 2012.

[20] J. Haddad and A. Shraiber, "Robust perimeter control design for an urban region," Transportation Research Part B: Methodological, vol. 68, pp. 315-332, 2014.

[21] C. F. Daganzo, "Urban gridlock: macroscopic modeling and mitigation approaches," Transportation Research Part B: Methodological, vol. 41, no. 1, pp. 49-62, 2007.

[22] Y. Wan, J. Cao, W. Huang, J. Guo, and Y. Wei, "Perimeter control of multiregion urban traffic networks with time- varying delays," IEEE Transactions on Systems, Man, and Cybernetics: Systems, vol. 50, no. 8, pp. 2795-2803, 2020.

[23] A. Kouvelas, M. Saeedmanesh, and N. Geroliminis, "Enhancing model-based feedback perimeter control with datadriven online adaptive optimization," Transportation Research Part B: Methodological, vol. 96, pp. 26-45, 2017.

[24] J. Haddad and B. Mirkin, "Coordinated distributed adaptive perimeter control for large-scale urban road networks," Transportation Research Part C: Emerging Technologies, vol. 77, pp. 495-515, 2017.

[25] M. Yildirimoglu, M. Ramezani, and N. Geroliminis, "Equilibrium analysis and route guidance in large-scale networks with MFD dynamics," Transportation Research Procedia, vol. 9, pp. 185-204, 2015.

[26] M. Yildirimoglu, I. I. Sirmatel, and N. Geroliminis, "Hierarchical control of heterogeneous large-scale urban road networks via path assignment and regional route guidance," Transportation Research Part B: Methodological, vol. 118, pp. 106-123, 2018.

[27] M. Ramezani, J. Haddad, and N. Geroliminis, "Dynamics of heterogeneity in urban networks: aggregated traffic modeling and hierarchical control," Transportation Research Part B: Methodological, vol. 74, pp. 1-19, 2015.

[28] I. I. Sirmatel and N. Geroliminis, "Economic model predictive control of large-scale urban road networks via perimeter control and regional route guidance," IEEE Transactions on Intelligent Transportation Systems, vol. 19, no. 4, pp. 11121121, 2017.

[29] K. An, Y.-C. Chiu, X. Hu, and X. Chen, "A network partitioning algorithmic approach for macroscopic fundamental diagram-based hierarchical traffic network management," IEEE Transactions on Intelligent Transportation Systems, vol. 19, no. 4, pp. 1130-1139, 2018.

[30] N. Geroliminis, J. Haddad, and M. Ramezani, "Optimal perimeter control for two urban regions with macroscopic fundamental diagrams: a model predictive approach," IEEE Transactions on Intelligent Transportation Systems, vol. 14, no. 1, pp. 348-359, 2013.

[31] S. Darmoul, S. Elkosantini, A. Louati, and L. Ben Said, "Multiagent immune networks to control interrupted flow at signalized intersections," Transportation Research Part C: Emerging Technologies, vol. 82, pp. 290-313, 2017.

[32] B. Chen, H. H. Cheng, and J. Palen, "Integrating mobile agent technology with multi-agent systems for distributed traffic detection and management systems," Transportation Research Part C: Emerging Technologies, vol. 17, no. 1, pp. 1-10, 2009.

[33] S. Kim, S. Tak, and H. Yeo, "Agent-based network transmission model using the properties of macroscopic fundamental diagram," Transportation Research Part C: Emerging Technologies, vol. 93, pp. 79-101, 2018.

[34] L. Leclercq, C. Parzani, V. L. Knoop, J. Amourette, and S. P. Hoogendoorn, "Macroscopic traffic dynamics with heterogeneous route patterns," Transportation Research Part C: Emerging Technologies, vol. 59, pp. 292-307, 2015.

[35] J. Wang, S. Peeta, X. He, and J. Zhao, "Combined multinomial logit modal split and paired combinatorial logit traffic assignment model," Transportmetrica A: Transport Science, vol. 14, no. 9, pp. 737-760, 2018.

[36] S. Kim, S. Tak, and H. Yeo, "Investigating transfer flow between urban networks based on a macroscopic fundamental diagram," Transportation Research Record: Journal of the Transportation Research Board, vol. 2672, no. 20, pp. 75-85, 2018. 
[37] J. Haddad, M. Ramezani, and N. Geroliminis, "Cooperative traffic control of a mixed network with two urban regions and a freeway," Transportation Research Part B: Methodological, vol. 54, pp. 17-36, 2013.

[38] S. Kim, S. Tak, D. Lee, and H. Yeo, "Distributed model predictive approach for large-scale road network perimeter control," Transportation Research Record: Journal of the Transportation Research Board, vol. 2673, no. 5, pp. 515-527, 2019.

[39] W.-X. Zhu and S. Li, "Study on discrete boundary-feedbackcontrol strategy for traffic flow based on macroscopic fundamental diagram," Physica A: Statistical Mechanics and Its Applications, vol. 523, pp. 1237-1247, 2019. 